\title{
REFERENCES
}

1. Eckford Cohen, Sums of an even number of squares in $G F\left[p^{n}, x\right]$. II, Duke Math. J. vol. 14 (1947) pp. 543-557.

2. Andre Weil, Numbers of solutions of equations in finite fields, Bull. Amer. Math. Soc. vol. 55 (1949) pp. 497-508.

DURE UnIVERSITY

\section{CONGRUENCES CONNECTED WITH THREE-LINE LATIN RECTANGLES}

L. CARLITZ

1. Introduction. In a recent paper [1], J. Riordan set up the recurrences

$$
K_{n}=n^{2} K_{n-1}+(n)_{2} K_{n-2}+2(n)_{3} K_{n-3}+k_{n},
$$

where $(n)_{r}=n(n-1) \cdots(n-r+1)$, and

$$
k_{n}+n k_{n-1}=-(n-1) 2^{n} ;
$$

here $K_{n}=K(3, n)$, the number of reduced three-line latin rectangles. He also proved the congruences

$$
k_{n+p} \equiv 2 k_{n}, \quad K_{n+p} \equiv 2 K_{n}(\bmod p),
$$

where $p$ is a prime $>2$.

In the present note we shall extend (1.3). We show first that for arbitrary $m$,

$$
k_{n+m} \equiv 2^{m} k_{n}, \quad K_{n+m} \equiv 2^{m} K_{n}(\bmod m) .
$$

More generally if we define

$$
\Delta f(n)=f(n+m)-2^{m} f(n), \quad \Delta^{\bullet} f(n)=\Delta^{0-1} f(n)
$$

for fixed $m \geqq 1$, then

$$
\Delta^{r} k_{n} \equiv 0 \equiv \Delta^{r} K_{n}\left(\bmod m^{r}\right)
$$

for all $r \geqq 1$.

2. Proof of (1.4). In (1.2) replace $n$ by $n+m$ so that

Presented to the Society, June 21, 1952; received by the editors April 16, 1952. 


$$
k_{n+m}+(n+m) k_{n+m-1}=-(n+m-1) 2^{n+m} .
$$

Comparison with (1.2) yields

$$
\left(k_{n+m}-2^{m} k_{n}\right)+n\left(k_{n+m-1}-2^{m} k_{n-1}\right)+m\left(k_{n+m-1}+2^{n+m}\right)=0
$$

or more briefly, using (1.5),

$$
\Delta k_{n}+n \Delta k_{n-1}+m\left(k_{n+m-1}+2^{n+m}\right)=0 .
$$

Clearly (2.1) implies

$$
\Delta k_{n} \equiv(-1)^{n} n ! \Delta k_{0}(\bmod m) .
$$

Since by (1.2)

$$
\Delta k_{0}=k_{m}-2^{m} k_{0} \equiv 2^{m}-2^{m} k_{0} \equiv 0(\bmod m),
$$

it follows at once that $\Delta k_{n} \equiv 0(\bmod m)$. This proves the first half of (1.4).

In the next place, using (1.1) we get

(2.2) $K_{n+m} \equiv n^{2} K_{n+m-1}+(n)_{2} K_{n+m-2}+2(n)_{8} K_{n+m-8}+k_{n+m}$,

so that

$$
\Delta K_{n} \equiv n^{2} \Delta K_{n-1}+(n)_{2} \Delta K_{n-2}+2(n)_{8} \Delta K_{n-8} .
$$

Since by (1.1)

$$
\begin{gathered}
K_{m} \equiv k_{m}, \quad K_{m+1} \equiv K_{m}+k_{m+1} \equiv k_{m}+k_{m+1} \equiv 0 \\
K_{m+2} \equiv 4 K_{m+1}+2 K_{m}+k_{m+2} \equiv 2 k_{m}+k_{m+2} \equiv 0
\end{gathered}
$$

(using the special values $k_{0}=1, k_{1}=-1, k_{2}=-2, K_{0}=1, K_{1}=K_{2}=0$ ), it follows that

$$
\Delta K_{0} \equiv \Delta K_{1} \equiv \Delta K_{2} \equiv 0(\bmod m) .
$$

Clearly (2.3) and (2.4) imply

$$
\Delta K_{n} \equiv 0(\bmod m)
$$

for all $n \geqq 0$. This completes the proof of (1.4).

3. Proof of (1.6). We shall require an extension of (2.1). Replacing $n$ by $n+m$, we get

$$
\Delta^{2} k_{n}+n \Delta^{2} k_{n-1}+2 m \Delta k_{n+m-1}=0,
$$

and it is then easy to get the general formula

$$
\Delta^{r} k_{n}+n \Delta^{r} k_{n-1}+r m \Delta^{r-1} k_{n+m-1}=0 \quad(n \geqq 0)
$$


for $r \geqq 2$. We now use (3.1) to prove

$$
\Delta^{r} k_{n} \equiv 0\left(\bmod m^{r}\right)
$$

$(r \geq 1)$.

Indeed we have already proved (3.2) for the value $r=1$. If then we assume (3.2) for the value $r-1,(3.1)$ implies

$$
\Delta^{r} k_{n} \equiv-n \Delta^{r} k_{n-1} \equiv(-1)^{n} n ! \Delta^{r} k_{0} .
$$

Now if we take $n=0$ in (3.1) we get

$$
\Delta^{r} k_{0}=-r m \Delta^{r-1} k_{m-1} \equiv 0,
$$

by the inductive hypothesis. Hence (3.3) reduces to (3.2). This proves the first half of (1.6).

We now prove

$$
\Delta^{r} K_{n} \equiv 0\left(\bmod m^{r}\right)
$$

By (2.5), (3.4) holds for $r=1$; we therefore assume that it holds for the value $r-1$. Now it follows from (1.1) that

$$
\begin{aligned}
\Delta^{r} K_{n}=n^{2} \Delta^{r} K_{n-1}+(n)_{2} \Delta^{r} K_{n-2} & +2(n)_{2} \Delta^{r} K_{n-3}+\Delta^{r} k_{n} \\
+r\left\{\left(\Delta n^{2}\right) \Delta^{r-1} K_{n+m-1}\right. & +\left(\Delta(n)_{2}\right)^{r-1} K_{n+m-2} \\
& \left.+2\left(\Delta(n)_{3}\right)^{r-1} K_{n+m-3}\right\}+\cdots
\end{aligned}
$$

for all $n \geqq 0$. Since $\Delta^{\bullet} n^{k} \equiv 0\left(\bmod m^{0}\right)$ for $k \geqq 0$, it is evident from (3.5) that we need merely examine $\Delta^{r} K_{n}$ for $n=0,1,2$. In the first place (3.5) implies $\Delta^{r} K_{0} \equiv 0\left(\bmod m^{r}\right)$ by the inductive hypothesis. Secondly for $n=1$, we see that $\Delta^{r} K_{1} \equiv \Delta^{r} K_{0} \equiv 0$, and for $n=2, \Delta^{r} K_{2} \equiv 4 \Delta^{r} K_{1}$ $+2 \Delta^{r} K_{0} \equiv 0$. Thus $\Delta^{r} K_{n} \equiv 0$ for all $n \geqq 0$. This completes the proof of (1.6).

\section{REFERENCE}

1. John Riordan, A recurrence relation for three-line latin rectangles, Amer. Math. Monthly vol. 59 (1952) pp. 159-162.

DUKR UNIVERSITY 